\title{
Secure Multi-Party Computation of Boolean Circuits with Applications to Privacy in On-Line Marketplaces
}

\author{
Seung Geol Choi ${ }^{1}$, Kyung-Wook Hwang ${ }^{2}$, Jonathan Katz ${ }^{1}$, \\ Tal Malkin ${ }^{2}$, and Dan Rubenstein ${ }^{2}$ \\ 1 University of Maryland \{sgchoi,jkatz\}@cs.umd.edu \\ 2 Columbia University $\{k w h w a n g @ e e, t a l @ c s$, danr@cs $\}$. columbia.edu
}

\begin{abstract}
Protocols for generic secure multi-party computation (MPC) generally come in two forms: they either represent the function being computed as a boolean circuit, or as an arithmetic circuit over a large field. Either type of protocol can be used for any function, but the choice of which protocol to use can have a significant impact on efficiency. The magnitude of the effect, however, has never been quantified.

With this in mind, we implement the MPC protocol of Goldreich, Micali, and Wigderson [13], which uses a boolean representation and is secure against a semi-honest adversary corrupting any number of parties. We then consider applications of secure MPC in on-line marketplaces, where customers select resources advertised by providers and it is desired to ensure privacy to the extent possible. Problems here are more naturally formulated in terms of boolean circuits, and we study the performance of our MPC implementation relative to existing ones that use an arithmetic-circuit representation. Our protocol easily handles tens of customers/providers and thousands of resources, and outperforms existing implementations including FairplayMP [3], VIFF [11], and SEPIA [7].
\end{abstract}

\section{Introduction}

Protocols for secure multi-party computation allow a set of parties $P_{1}, \ldots, P_{n}$ to compute some function of their inputs in a distributed fashion, while revealing nothing to a coalition of corrupted parties about any honest party's input (or any group of honest parties' inputs), beyond what is implied by the output. Seminal results in cryptography dating to the 1980 s $[30,13,12]$ show that any polynomial-time function can be computed securely in the presence of coalitions of up to $n-1$ corrupted parties. For many years, the perception was that these were to be viewed as purely theoretical results with little practical relevance. This changed (to some extent) with the advent of Fairplay [24], an implementation of Yao's protocol for secure two-party computation that demonstrated for the first time that generic protocols were within the realm of feasibility. Since then, several implementations of generic secure two-party and multi-party computation protocols have been developed $[3,22,5,11,26,7,15,23]$, and this is currently an active area of research. 
In this work our focus is on generic protocols for secure multi-party computation (MPC) in the semi-honest setting. (In the semi-honest setting, parties are assumed to follow the protocol but coalitions of malicious parties may attempt to learn additional information from the joint transcript of their execution of the protocol. By "generic" we mean protocols that can be used to securely compute arbitrary functions.) There are, broadly speaking, two approaches taken by protocols for secure MPC: they either represent the function being computed as a boolean circuit, or as an arithmetic circuit over a (cryptographically) large field $^{1} \mathbb{F}$. Although any function $f$ can be computed using either type of protocol, the choice of representation affects the size of the circuit implementing $f$, and hence the overall efficiency of a secure protocol for computing $f$. The magnitude of the effect, however, has never been measured experimentally.

Most existing implementations of secure MPC rely on an arithmetic-circuit representation, with ShareMind [4], VIFF [11], and SEPIA [7] serving as prominent examples. We are aware of only one existing implementation of secure MPC (namely, FairplayMP [3]) using boolean circuits. As we will see, for certain problems a boolean-circuit representation is more natural, and so it is important to have protocols of both types available. Indeed, the motivation for our work came from trying to apply secure MPC to privacy-preserving computation in on-line marketplaces, where customers select resources advertised by providers and it is desired to ensure privacy to the extent possible. (See the following section for details.) In doing so, we found that existing implementations of secure MPC were unsuitable or too inefficient for our purposes. Moreover, all the MPC implementations mentioned above assume an honest majority, even though resilience against an arbitrary number of corruptions is known to be attainable.

\subsection{Our Contributions}

We implemented the classical MPC protocol of Goldreich, Micali, and Wigderson [13] (the GMW protocol), which uses a boolean-circuit representation for the function being computed and is secure against a semi-honest adversary controlling any number of corrupted parties. In our implementation, described in Section 2, we employ several optimizations to improve efficiency. Our code is publicly available ${ }^{2}$ and we expect that, as with other systems, it will be useful in future work on privacy-preserving distributed computation.

With our system in place, any privacy-preserving multi-party computation can be solved, in principle, by defining an appropriate circuit for the task at hand. We designed circuits addressing three different (but related) problems in the context of on-line marketplaces where, generally speaking, providers advertise resources to be selected and subsequently utilized by customers, and the purpose of the marketplace is to match customers with providers in a way that optimizes some value under a certain set of constraints. We look at the following examples:

\footnotetext{
${ }^{1}$ Of course, a boolean circuit can be viewed as a circuit over the field $\mathbb{F}=G F(2)$. The distinction is that protocols using arithmetic circuits require $1 /|\mathbb{F}|$ to be negligible in order for security and correctness to hold.

${ }^{2}$ http://www. ee.columbia.edu/ ${ }^{\sim}$ kwhwang/projects/gmw.html
} 
- P2P content-distribution services $[9,18]$ provide a marketplace where content is the resource, and providers advertise availability of content at peers. Here, a customer may want to determine which peer hosting the desired content is the best choice (e.g., closest, or having minimal delay) for retrieving that content.

- In cloud computing providers are cloud platforms (e.g., Amazon EC2, Microsoft Azure, etc.), resources are the services (e.g., storage, bandwidth, or processing) offered by each provider, and customers want to find the provider(s) offering services matching their needs at the cheapest price $[1,8$, $29,27,28,20]$.

- A mobile social network can be viewed as a marketplace where users are both customers and resources, and the provider helps users locate and connect to other users who share similar interests.

Formal definitions of the problems in each of the above settings are given in Section 3, and we describe optimized circuits solving each of them in the full version of this paper [10]. For these problems, we find that it significantly helps to be able to work with boolean circuits rather than arithmetic circuits.

In Section 4 we evaluate the performance of our MPC protocol as applied to one of the above problems. (Since they have similar circuits, the other two problems should display similar results.) Our results shows that our protocol can be used to efficiently and securely implement a distributed marketplace with tens of providers/customers and thousands of resources over a wide-area network. Our implementation outperforms systems such as VIFF [11] and SEPIA [7], in part because we use boolean circuits rather than arithmetic circuits as those systems do. ${ }^{3}$ Another advantage of our protocol is that it provides security against any number of corruptions, whereas the cited implementations $[3,4,11,7]$ all require an honest majority.

\subsection{Other Related Work}

There are several existing implementations of secure two-party computation [24, $22,26,14,15,23]$. These are all specific to the two-party setting and do not yield protocols for three or more parties. Interestingly, and in contrast to other multiparty implementations $[3,11,7]$ that only handle three or more parties, the GMW protocol we implement can handle any number of parties $n \geq 2$. For the twoparty case, however, we expect our implementation to be roughly a factor of two slower than the best available system [15].

Other implementations of secure multi-party computation, besides those discussed above, include $[6,4,17]$. The code for SIMAP $[6]$ is not publicly available, and anyway SIMAP appears to be superseded by VIFF. Sharemind [4] handles only the three-party setting, assuming at most one semi-honest corruption. The work of Jakobsen et al. [17] achieves resilience against an arbitrary number of

\footnotetext{
${ }^{3}$ FairplayMP [3] also uses boolean circuits, but did not support multiple input values per party and crashed on the input sizes used. See Section 4 for further discussion. We do not compare to ShareMind since that system only supports 3-party computation.
} 
malicious corruptions. Their implementation is based on arithmetic circuits and has worse performance than VIFF (though with better resilience).

\section{MPC Implementation}

We provide an overview of the GMW protocol and details of our implementation. The GMW protocol provides security against a semi-honest adversary corrupting any number of parties. (We refer to [12] for formal definitions of security.) Assuming semi-honest behavior is reasonable in settings where the codebase is difficult to change without detection, where software attestation can be used to convince other parties that correct software is being run, or where parties are trusted but must ensure secrecy of data for policy reasons or because of concerns about future break-ins.

\subsection{Overview of the GMW Protocol}

1-out-of-4 oblivious transfer. Oblivious transfer (OT) is a key building block of the GMW protocol. A 1-out-of-4 OT protocol is a two-party protocol in which there is a sender holding values $\left(x_{0}, x_{1}, x_{2}, x_{3}\right)$ and a receiver holding an index $i \in\{0, \ldots, 3\}$; the receiver learns $x_{i}$, but neither the sender nor the receiver learn anything else; i.e., the receiver learns nothing about any other values held by the sender, and the sender learns nothing about the receiver's index.

Details of our OT implementation are given in Section 2.2.

The GMW protocol. The GMW protocol assumes the function $f$ to be computed is represented as a boolean circuit consisting of XOR and AND gates or, equivalently, gates for addition and multiplication modulo 2 . Let $n$ denote the number of parties. In the GMW protocol the parties maintain random $n$-out-of- $n$ shares $\left(s_{w 1}, \ldots, s_{w n}\right)$ of the value $s_{w}$ on each wire $w$ in the circuit; that is, party $P_{i}$ holds share $s_{w i}$ and all shares are random subject to $s_{w}=\bigoplus_{i} s_{w i}$. Setting up such shares on the input wires is easy: party $P_{i}$ with input $s_{w}$ on wire $w$ chooses random $s_{w j}$ for $j \neq i$, sends $s_{w j}$ to $P_{j}$, and locally sets $s_{w i}=s_{w} \oplus\left(\bigoplus_{j \neq i} s_{w j}\right)$. Shares on internal wires of the circuit are then computed inductively in the following way:

XOR gates. Say $w$ is the output wire of an XOR gate with input wires $u$ and $v$, and the parties have shares $\left(s_{u 1}, \ldots, s_{u n}\right)$ and $\left(s_{v 1}, \ldots, s_{v n}\right)$ of $s_{u}$ and $s_{v}$, respectively. Then each party $P_{i}$ locally computes $s_{w i}=s_{u i} \oplus s_{v i}$, and one can observe that $\left(s_{w 1}, \ldots, s_{w n}\right)$ is a valid sharing of $s_{w}=s_{u} \oplus s_{v}$.

AND gates (cf. [12]). Say $w$ is the output wire of an AND gate with input wires $u$ and $v$, and the parties have shares $\left(s_{u 1}, \ldots, s_{u n}\right)$ and $\left(s_{v 1}, \ldots, s_{v n}\right)$ of $s_{u}$ and $s_{v}$, respectively. Note that

$$
s_{w}=s_{u} \cdot s_{v}=\left(\sum_{i=1}^{n} s_{u i}\right) \cdot\left(\sum_{i=1}^{n} s_{v i}\right)=\sum_{i=1}^{n} s_{u i} s_{v i}+\sum_{i<j}\left(s_{u i} s_{v j}+s_{u j} s_{v i}\right) .
$$


Each party $P_{i}$ can compute $s_{u i} s_{v i}$ locally. As for the remaining term, each pair of parties $P_{i}, P_{j}$ computes a random additive share of $s_{u i} s_{v j}+s_{u j} s_{v i}$ in the following way. $P_{j}$ chooses a random bit $c_{j}^{\{i, j\}}$, and computes four values

$$
c_{j}^{\{i, j\}}, \quad c_{j}^{\{i, j\}} \oplus s_{v j}, \quad c_{j}^{\{i, j\}} \oplus s_{u j}, \quad c_{j}^{\{i, j\}} \oplus s_{v j} \oplus s_{u j}
$$

corresponding to the four possible values of $P_{i}$ 's shares $s_{u i}, s_{v i}$. Party $P_{i}$ then acts as a receiver in 1-out-of-4 OT, with index determined by the actual values of its shares $s_{u i}, s_{v i}$, to obtain the appropriate value from $P_{j}$ that we denote by $c_{i}^{\{i, j\}}$. Note that $c_{i}^{\{i, j\}}+c_{j}^{\{i, j\}}=\left(s_{u i} s_{v j}+s_{u j} s_{v i}\right)$. Finally, each party $P_{i}$ computes

$$
s_{w i}=s_{u i} s_{v i}+\sum_{j \neq i} c_{i}^{\{i, j\}} \text {. }
$$

It can be verified that $\left(s_{w 1}, \ldots, s_{w n}\right)$ is a (random) sharing of $s_{w}=s_{u} \cdot s_{v}$.

Evaluation of XOR gates is essentially free, whereas evaluating AND gates requires $\left(\begin{array}{l}n \\ 2\end{array}\right)$ invocations of 1 -out-of- 4 oblivious transfer.

Once a sharing $\left(s_{w 1}, \ldots, s_{w n}\right)$ of an output wire $w$ is obtained, the value $s_{w}$ can be reconstructed by having each party privately send its share to all other parties. It is also possible for only some specific party to learn a given output value by sending shares to that party only. We note that this is the only step in the protocol where private channels are needed, and then only if more than one party is to learn a given output value.

\subsection{Oblivious-Transfer Protocols}

As noted in the previous section, oblivious transfer is a key building block of the GMW protocol; it is also the most computationally expensive part of the proto$\mathrm{col}$, since it is the only part of the protocol that relies on public-key techniques. As described above, the GMW protocol requires one invocation of 1-out-of-4 OT per pair of parties each time an evaluation of an AND gate is performed, and so $m$ executions of OT (per pair of parties) to evaluate a circuit containing $m$ AND gates. We can improve the overall efficiency, however, using two techniques:

- Using OT pre-processing [2], each pair of parties can perform $m$ oblivious transfers on random inputs at the outset of the protocol, and then (very efficiently) use the pre-computed values thus obtained to achieve the functionality of oblivious transfer on their actual inputs when evaluating an AND gate. Thus, all the oblivious transfers that will be needed throughout the entire protocol can be run in parallel at the beginning of the protocol.

- Using $O T$ extension $[16,21]$, it is possible to achieve the functionality of $m$ invocations of 1-out-of- 4 OT at essentially the cost of $k$ invocations of 1-outof- 4 OT of $m$-bit strings, where $k$ is a statistical security parameter. (More precisely, the marginal cost for each additional OT is just a small number of hash computations.) Security here is based on the assumption that the hash function is correlation robust [16]. 


\section{$k$ parallel invocations of 1-out-of-4 OT}

Let $g, \mathbb{G}$, and $q$ be fixed, where $\mathbb{G}$ is a cyclic group of prime order $q$, and $g$ is a generator of $\mathbb{G}$. Let $H:\{0,1\}^{*} \rightarrow\{0,1\}^{m}$ be a hash function.

InPUTS. S holds $\left\{\left(x_{0}^{j}, x_{1}^{j}, x_{2}^{j}, x_{3}^{j}\right)\right\}_{j \in[k]}$ with $x_{i}^{j} \in\{0,1\}^{m} . \mathbf{R}$ holds $\left(r_{1}, \ldots, r_{k}\right)$ where $r_{j} \in\{0, \ldots, 3\}$.

THE PROTOCOL.

1. $\mathbf{S}$ chooses $\alpha \leftarrow \mathbb{Z}_{q}$ and computes $c_{0}=g^{\alpha}$, and also chooses $c_{1}, c_{2}, c_{3} \leftarrow \mathbb{G}$. It sends $c_{0}, \ldots, c_{3}$ to $\mathbf{R}$.

For $j \in[k]$ the parties do:

2. $\mathbf{R}$ chooses $\beta_{j} \leftarrow \mathbb{Z}_{q}$. If $r_{j}=0$ then it sets $d_{j}=g^{\beta_{j}}$; else, it sets $d_{j}=$ $c_{r_{j}} / g^{\beta_{j}}$. Finally, $\mathbf{R}$ sends $d_{j}$ to $\mathbf{S}$.

3. $\mathbf{S}$ computes $e_{0}=d_{j}^{\alpha}$ and $e_{i}=\left(c_{i} / d_{j}\right)^{\alpha}$ for $i \in\{1,2,3\}$. Then $\mathbf{S}$ sends $\bar{x}_{i}^{j}=H\left(e_{i}, j, i\right) \oplus x_{i}^{j}$ to $\mathbf{R}$ for $i \in\{0, \ldots, 3\}$.

4. $\mathbf{R}$ computes $c_{0}^{\beta_{j}}=e_{r_{j}}$, and then outputs $x_{r_{j}}^{j}=\bar{x}_{r_{j}}^{j} \oplus H\left(e_{r_{j}}, j, r_{j}\right)$.

Fig. 1. The Naor-Pinkas OT protocol.

Combining these optimizations, each pair of parties needs only run $k$ (parallel) invocations of some "base" OT protocol (for $m$-bit strings) at the outset. These can be converted to $m \gg k$ OT executions (on bits) using OT extension; these $m$ "pre-processed" OTs can then be used, as needed, during the rest of the protocol. It remains only to specify the "base" 1-out-of-4 OT protocol we use. We take as our base OT protocol the one by Naor and Pinkas [25], secure under the decisional Diffie-Hellman (DDH) assumption in the random-oracle model. Their protocol (actually, a version implementing $k$ parallel executions of their protocol) is described in Figure 1 for completeness.

\subsection{Implementation Details}

We implemented the GMW protocol in $\mathrm{C}++$. Our implementation takes as input a file containing a description of a boolean circuit for the function $f$ of interest. (All parties are assumed to be running with identical copies of the circuit.) See Section 2.4 for an example. Unlike FairplayMP [3], we do not provide a mechanism for compiling a high-level language into a boolean circuit.

Parallelism. Nowadays, it is common for computers to have multiple cores. We use multi-threaded programming so as to take advantage of the available parallelism. In particular, each OT execution is performed by a separate thread. In the OT extension protocol, we optimize execution time by having parties send values as soon as they are computed, rather than waiting for the other party to finish sending. (This does not affect security, since this occurs at fixed times that are independent of the parties' inputs and we assume semi-honest behavior.)

Random oracle. We use SHA-1 to implement a random oracle $H$ with arbitrary output length by defining

$$
H(M)=\text { SHA-1 }(\text { seed }, 0) \| \text { SHA-1 }(\text { seed }, 1) \| \cdots,
$$


where seed $=$ SHA-1 $(M)$. Note that seed need only be computed once. We use the SHA-1 implementation of PolarSSL (http://polarssl.org).

Oblivious transfer. For our base OT protocol we use the Naor-Pinkas protocol (see Figure 1) with group $\mathbb{G} \subset \mathbb{Z}_{p}$ of prime order $q$, and $p=2 q+1$ with $p$ prime. In our default implementation, $p$ is a 1024-bit integer. We modified the modular-arithmetic module of NTL (http: //www . shoup. net/ntl) to be threadsafe, and used it to implement the base OT protocol. Recall we use OT extension to improve efficiency. By default, we use statistical security parameter $k=80$ in our implementation. Messages are transmitted in chunks of reasonable size to obtain a balance between the idle time and the number of socket calls.

\subsection{Circuit Example}

Our implementation of the GMW protocol takes as input (at each party running the protocol) three files that contain configuration information, the input of the party in question, and a description of a boolean circuit for the function $f$ of interest. (All parties are assumed to be running with identical copies of the circuit.) In Figure 2 we show an example circuit along with its description using our representation.

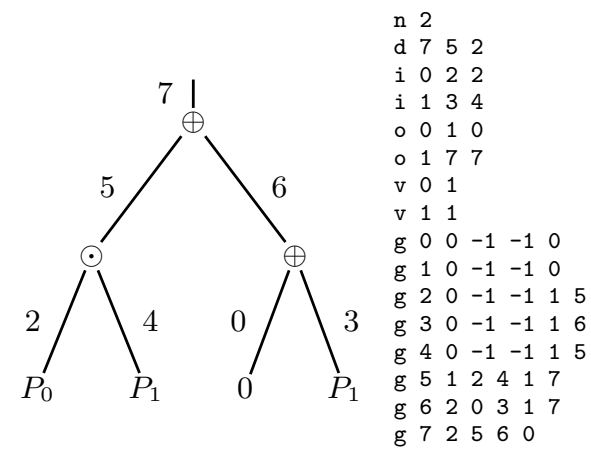

Fig. 2. Circuit Example The circuit description uses the following format:

- The first line of the file has the form $\mathrm{n} X$, where $X$ denotes the number of parties participating in the protocol.

- The second line of the file contains a d followed by the total number of wires in the circuit, the number $w$ of the first non-input wire (i.e., wires 0 to $w-1$ are input wires), and the number of XOR gates in the circuit.

- For each party, there is a line containing an i followed by the party's id, the number of the first input wire belonging to that party, and the number of the last input wire belonging to that party. (We assume wires are numbered such that every party provides inputs on a consecutive set of wires.)

- For each party, there is a line in the file containing an o followed by the party's id, the number of the first output wire belonging to that party, and the number of the last output wire belonging to that party. (We assume wires are numbered such that every party receives outputs on a consecutive set of wires.) If a party receives no output, the number of the last output wire for that party is set to 0 .

- For each party, there is a line in the file containing a $\mathrm{v}$ followed by the party's id and then an integer denoting the number of bits that should be used to represent each item in that party's input file. (E.g., if the input file of party 1 contains a ' 4 ' then this value will be represented as the 3-bit integer ' 100 ' if this line of the file is ' $v 13$ ', but will be represented as the 5-bit integer 
'00100' if this line of the file is ' $\mathrm{v} 15$ '.) Each bit in the ultimate representation of the integer will correspond to one of the input wires of the party.

- The remaining lines of the file describe the gates in the circuit. For each gate, we list (a) the number of the output wire of this gate (which also serves as the gate id); (b) the gate type, which can be either input (0), AND (1), or XOR (2); (c) the numbers of the left and right input wires (set to -1 if these are input gates); and (d) the out-degree of the gate. If the out-degree is nonzero, then the ids of the gates that receive the output of the current gate are listed. Gate ids 0 and 1 are reserved for the constants 0 and 1, respectively.

\section{Problem Definitions}

We introduce three problems in the context of on-line marketplaces where, generally speaking, providers advertise resources to be selected and subsequently utilized by customers, and the function of the marketplace is to match customers with providers so as to optimize some value under a certain set of constraints. As highlighted in the Introduction, we look at examples in the settings of $\mathrm{P} 2 \mathrm{P}$ content distribution, cloud computing, and mobile social networks.

As a toy example, consider a customer who wishes to buy a car (resource) from one of several dealers (providers). The customer is interested in several different models of cars (but not all models); the different providers offer a variety of models (not all of which interest the customer); and each provider prices each model independently. The customer wishes to find an acceptable car at the lowest cost, without revealing the set of models he or she is interested in; the providers do not want to reveal their prices. Secure MPC allows the customer to learn the identity of a provider selling an acceptable model at the lowest price, with the customer learning no other prices (or which models are sold by each provider), and with the providers learning only of the customer's willingness to buy some particular model at the given price.

More formally, let $R$ be some set of resources. The input of each provider $P_{i}$ is a collection of values for the resources in some subset $R_{i} \subseteq R$; i.e., $P_{i}$ 's input is of the form $\left\{v_{r}^{i}\right\}_{r \in R_{i}}$. (If desired, each $P_{i}$ could just use some default value $v_{r}^{i}=\perp$ for $r \notin R_{i}$; in that case, we may simply write $P_{i}$ 's input as $\left\{v_{r}^{i}\right\}_{r \in R}$.) We look at marketplaces where the computation can be broken into the following two steps, which will be executed as a single secure computation (so only the final output is revealed, not the intermediate results after the first step):

1. For each provider $P_{i}$ and resource $r \in R_{i}$, compute a scoring function $\mathrm{sc}_{r}^{i}=$ Score $\left(i, r, v_{r}^{i}, x_{n}\right)$, where $x_{n}$ denotes the private input of the customer. (In the running toy example, each model is scored by its offered price if the model is of interest to the customer, and by $\infty$ otherwise.)

2. Next, apply a best-match function $\mathcal{B}$ to the set of $\mathrm{sc}_{r}^{i}$ values to obtain a result that is given to the customer. (In the toy example, $\mathcal{B}$ outputs $\left(i, r, \mathrm{sc}_{r}^{i}\right)$ with minimum $\mathrm{sc}_{r}^{i}$.) 
We allow the scoring function to be arbitrary. For the best-match function we consider two possibilities: either $\mathcal{B}$ returns a single $(i, r)$ maximizing/minimizing $\mathrm{sc}_{r}^{i}$ (with ties broken arbitrarily, and with or without including $\mathrm{sc}_{r}^{i}$ as part of the output), or $\mathcal{B}$ returns the set of all $(i, r)$ for which the score $\mathrm{sc}_{r}^{i}$ is greater/lower than some threshold. In the following subsections we instantiate this general framework in several specific scenarios.

\subsection{P2P Content-Distribution Services}

In our P2P content-distribution setting, content is replicated across various $\mathrm{P} 2 \mathrm{P}$ servers or source peers (such as seeders) whose pairwise communications are measured (and perhaps even controlled) by network providers such as ISPs. Before a peer starts downloading content, he or she would like to find out the best source peer (with respect to network bandwidth, end-to-end delays, throughput, and so on) from which to receive the content.

Here the providers are the ISPs and the resources are the source peers themselves (which for simplicity we identify with their indices). Let $R$ be the set of source peers, with $|R|=k$. We assume that the ISP to which each source peer is bound is public knowledge, so ISP $P_{i}$ is associated with some set of peers $R_{i}$. The input of each ISP/provider $P_{i}$ is the measured bandwidth $v_{r}^{i}$ for each peer/resource $r \in R_{i}$. The customer knows which peers have a replica of the item it wishes to retrieve, and holds as secret input a vector $x_{n}=\left(b_{1}, \ldots, b_{k}\right)$ where $b_{r}=1$ iff peer/resource $r$ has the desired content, and $b_{r}=0$ otherwise. The objective is for the customer to find the best (e.g., highest-bandwidth) peer among those holding the desired content, without revealing which source peers have the content; the ISPs do not want to reveal the bandwidth of their peers.

Here the scoring function can be defined as:

$$
\text { Score }\left(i, r, v_{r}^{i},\left(b_{1}, \ldots, b_{k}\right)\right)=\left\{\begin{array}{ll}
v_{r}^{i} \text { if } b_{r}=1 \\
0 & \text { otherwise }
\end{array},\right.
$$

and the best-match function $\mathcal{B}$ returns $i, r$ maximizing sc ${ }_{r}^{i}$. (In fact, it suffices to return $r$ here since the provider to which $r$ is bound is irrelevant and anyway known.)

\subsection{Cloud Computing}

In this setting, providers offer various service packages and the customer wants to select the service package meeting its needs at the lowest available price. The service packages offered by the providers are the resources here, and each such resource $r$ has a value $v_{r}=\left(q_{r}, p_{r}\right)$ that is composed of its service quality $q_{r}$ and price $p_{r}$. (For simplicity, we treat service quality as a one-dimensional quantity, e.g., CPU cycles. Our treatment can easily be generalized.) The customer holds input $(q, p)$, where $q$ represents a minimum acceptable service quality and $p$ is a maximum budget. Two scenarios can be considered: either the customer wants to find the cheapest resource $r$ with $q_{r} \geq q$, or the highest-quality resource $r$ with $p_{r} \leq p$; each of these cases is treated below. In either case, the customer 
never reveals its budget or its service requirements to any of the providers, nor do the providers reveal to the customer (or to each other) what service packages they are offering.

Lowest-price selection. In this formulation, the customer seeks the package that satisfies its requirements at the lowest price. Here we may define the scoring function as:

$$
\text { Score }\left(\left(i, r, q_{r}^{i}, p_{r}^{i}\right),(q, p)\right)=\left\{\begin{array}{l}
p_{r}^{i} \text { if } q_{r}^{i} \geq q \text { and } p_{r}^{i} \leq p \\
\infty \text { otherwise }
\end{array} .\right.
$$

The best-match function $\mathcal{B}$ returns an $i, r$ minimizing $\mathrm{sc}_{r}^{i}$.

Highest-quality selection. Here the customer seeks the package that meets its budget while giving the highest quality service. Now we may define the scoring function as:

$$
\text { Score }\left(\left(i, r, q_{r}^{i}, p_{r}^{i}\right),(q, p)\right)=\left\{\begin{array}{l}
q_{r}^{i} \quad \text { if } q_{r}^{i} \geq q \text { and } p_{r}^{i} \leq p \\
-\infty \text { otherwise }
\end{array}\right.
$$

and the best-match function returns an $i, r$ maximizing $\mathrm{sc}_{r}^{i}$.

\subsection{Mobile Social Networks}

Here we consider a scenario where a user in a social network wants to identify nearby users who share common interests. Now the resources and providers are just the set $R$ of all users (and the customer is one of the users as well), and the value of each "resource" (i.e., user) is that user's location and set of interests.

We assume that each user knows only about its own location and interests. Thus for each $r \in R$ we define $v_{r}^{r}=\left(\ell_{r}, H_{r}\right)$, where $\ell_{r}$ is the location of user $r$, and $H_{r}$ is the set of that user's interests (perhaps represented as a bit-vector). The customer's input consists of $(\ell, H, \delta)$ where $\ell$ is the location of the customer, $H$ is the set of interests she wants a potential match to share, and $\delta$ is the distance radius in which she wants to search. We consider a few alternatives for what the customer wants as output.

Find all close matches. In this formulation, the customer wants to find all users within distance $\delta$ who share interests $H$. We may then define

$$
\text { Score }\left(\left(r, \ell_{r}, H_{r}\right),(\ell, H, \delta)\right)=\left\{\begin{array}{l}
1 \text { if } H \subseteq H_{r} \text { and }\left|\ell_{r}-\ell\right| \leq \delta \\
0 \text { otherwise }
\end{array},\right.
$$

and the best-match function returns the set of all $r$ such that $\mathrm{sc}_{r}^{r}=1$.

Find closest match. Here the customer wants to find the closet user who matches her interests. Now, define

$$
\text { Score }\left(\left(r, \ell_{r}, H_{r}\right),(\ell, H, \delta)\right)= \begin{cases}\left|\ell_{r}-\ell\right| & \text { if } H \subseteq H_{r} \text { and }\left|\ell_{r}-\ell\right| \leq \delta \\ \infty & \text { otherwise }\end{cases}
$$

The best-match function returns an $r$ minimizing $\mathrm{sc}_{r}^{r}$. 


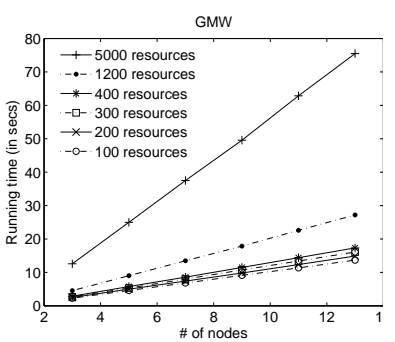

(a) GMW

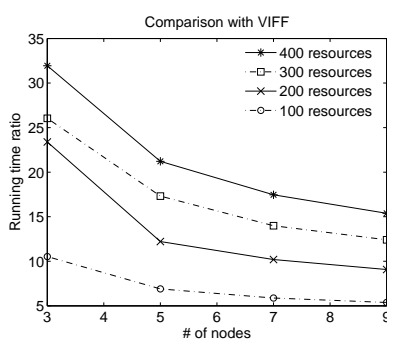

(b) $\frac{\text { VIFF }}{\text { GMW }}$

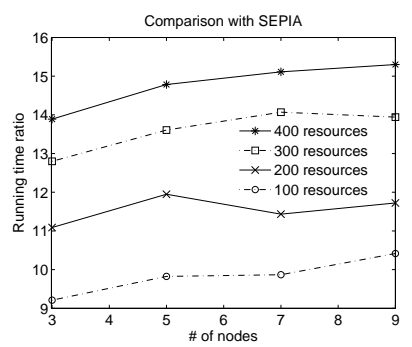

(c) $\frac{\text { SEPIA }}{\text { GMW }}$

Fig. 3. Running times in a LAN.

Find best resource. Now the customer would like to obtain the resource within radius $\delta$ that shares as many interests as possible. We thus define

$$
\text { Score }\left(\left(\ell_{r}, H_{r}\right),(\ell, H, \delta)\right)=\left\{\begin{array}{ll}
\left|H_{r} \cap H\right| & \text { if }\left|\ell_{r}-\ell\right| \leq \delta ; \\
-\infty & \text { otherwise }
\end{array},\right.
$$

and the best-match function returns $r$ maximizing $\mathrm{sc}_{r}^{r}$.

\subsection{Boolean-Circuit Constructions}

We construct appropriate boolean circuits solving each of the problems described above. Since XOR gates are essentially "free" to evaluate in the GMW protocol, whereas evaluating each AND gate requires cryptographic computations, we aimed to minimize the number of AND gates in the circuits. Due to lack of space, descriptions of our circuits are given in the full version [10].

\section{Performance Evaluation}

We evaluate the performance of our implementation in both a localarea network (LAN) and a wide-area network (using PlanetLab, see http://www.planet-lab.org/), and compare it to existing systems for secure MPC. In our experiments we consider only the P2P content-distribution problem formulated in Section 3.1 with 16-bit integer representation (i.e., $\ell=16$ ), but since circuits for the other two problems are similar (in terms of both circuit depth and the number of AND gates), we expect the results to be similar for those problems as well. We let GMW refer to our implemented protocol for this problem, obtained by applying our GMW implementation to the circuit (described in the full version [10]). All reported measurements are based on averages over 10 runs of the experiment in question.

\subsection{Local-Area Network}

Our first set of experiments is performed in a cluster consisting of multiple Linux host nodes, each containing two Intel Xeon 2.80GHz CPUs and 4GB RAM. We 


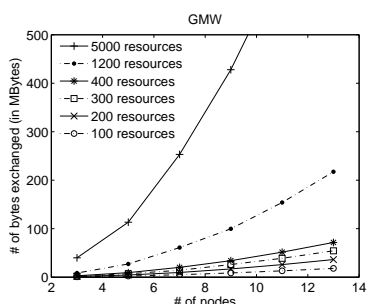

(a) GMW

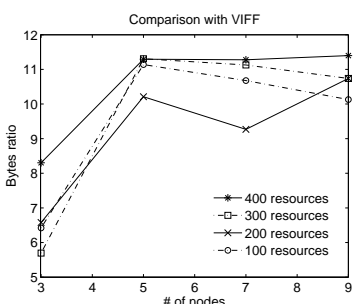

(b) $\frac{\text { VIFF }}{\text { GMW }}$

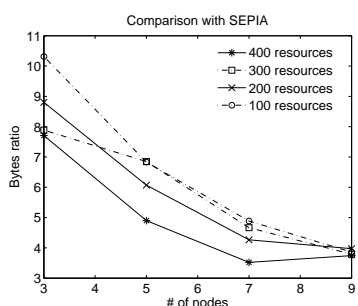

(c) $\frac{\text { SEPIA }}{\text { GMW }}$

Fig. 4. Total bytes transferred among all nodes.

use one host per participant in the protocol, so an experiment with $n$ providers involves an $(n+1)$-party multi-party computation on $n+1$ host machines. We set up our experiments so the customer chooses half the resources offered by each provider to be "of interest". Note that the client's inputs do not affect performance, since the same underlying circuit is evaluated regardless of the customer's input; indeed, if performance were affected by the customer's input then the protocol could not be secure.

We ran experiments using from 3 to 13 host nodes, and 100 to 5,000 resources. (This represents the aggregate offered by all providers.) For this problem, the number of AND gates being evaluated depends on the number of resources only (it is independent of the number of nodes), and ranges from about 5,500 AND gates (for 100 resources) to roughly 305,000 AND gates (for 5,000 resources). The running time is plotted in Figure 3(a), and the total bandwidth (between all parties) is shown in Figure 4(a).

For a fixed number of resources, the bandwidth grows quadratically with the number of nodes; this is because each pair of parties communicates for every AND gate being evaluated. The running time scales linearly with the number of nodes since all nodes work in parallel, and the work per node increases in direct proportion to the number of other nodes with which it communicates. Although difficult to see from the plots, for a fixed number of parties the running time and bandwidth increase roughly linearly in the number of resources $k$; this is because the size of the circuit grows roughly linearly in $k$ (actually, it grows as $k \log k$ but the logarithmic term is difficult to detect).

We also measured the marginal time to evaluate a single AND gate (i.e., the time required to evaluate one additional AND gate, once the number of AND gates is large). We use marginal time because there is a fixed cost for the initial oblivious transfers performed by the parties, but then oblivious-transfer extension is used to get additional OTs at much lower cost (see Section 2.2). The marginal cost per AND gate ranged from $50 \mu \mathrm{s}$ (for 3 parties) to $340 \mu \mathrm{s}$ (for 13 parties).

Comparison to existing work. We applied other existing implementations of secure MPC to the same problem. Unfortunately, despite contacting the authors we were unable to get a working implementation using FairplayMP [3] since we found that it did not support providing users with multiple inputs, and it 
would crash (when parties were provided with a single input) on inputs more than 16 bits long. ${ }^{4}$ We were able to compare our protocol with implementations in (the semi-honest version of) VIFF [11] and SEPIA [7]. We ran both VIFF and SEPIA over insecure (i.e., non-SSL-protected) channels even though private channels would be needed to ensure security against an eavesdropping adversary for those protocols. (In contrast, for GMW a secure channel is not needed if only one party learns the output; when multiple parties learn the output, only the finalround messages need to be encrypted.) In SEPIA, parties provide their inputs to "privacy peers" that run a secure-computation protocol on their behalf; when we refer to "nodes" in SEPIA we mean the number of privacy peers.

In contrast to GMW, VIFF and SEPIA utilize arithmetic circuits where each wire carries an element of a large field $\mathbb{F}$ (with $\log |\mathbb{F}| \approx 64$ in each case), and gates perform addition or multiplication in $\mathbb{F}$. (Similar to the GMW case, addition is essentially "for free" whereas multiplication is "expensive".) The boolean circuit we used for GMW is easily adapted for VIFF/SEPIA as follows (see the full version of this paper [10] for further details):

- Boolean values can be represented as elements of $\mathbb{F}$. Boolean operations can be performed as $\operatorname{AND}(a, b)=a b$ and $\operatorname{XOR}(a, b)=a+b-2 a b$ (where computations are in $\mathbb{F}$ ), so long as $a, b \in\{0,1\}$. (Note, however, that both operations involve a multiplication in $\mathbb{F}$.)

- $\ell$-bit integers can be represented as elements of $\mathbb{F}$, since $|\mathbb{F}| \gg 2^{\ell}$ for the value of $\ell$ we use. Because of this, addition and subtraction gates are now trivial to implement since they correspond exactly to addition and subtraction over $\mathbb{F}$.

- VIFF and SEPIA already provide circuits for performing comparisons.

In Figures 3(b) and 3(c) (resp., Figures 4(b) and 4(c)) we compare GMW's running time (resp., bandwidth utilization) to that of VIFF and SEPIA. Since the running times of VIFF and SEPIA are comparatively long, we only ran experiments with up to 400 resources and up to 9 nodes. In those ranges of the parameters, GMW completes in under 20 seconds while VIFF and SEPIA take an order of magnitude longer; the relative performance of GMW becomes even better as the number of resources is increased. The results demonstrate that our implementation scales significantly better, and is more efficient, than prior implementations. Recall also that GMW withstands a larger number of corruptions than either VIFF or SEPIA.

\subsection{Wide-Area Network}

We explored the effects of communication latency by running our implementation of the GME protocol in a wide-area network (WAN) using PlanetLab (http://www.planet-lab.org/). In the PlanetLab settings we explored, the

\footnotetext{
${ }^{4}$ We did compare the performance of GMW to FairplayMP for 5-party computation of a "toy" circuit consisting of a depth- $d$ full binary tree of AND gates. With $d=12$, GMW ran about 20 times faster than FairplayMP. FairplayMP crashes on circuits with $d>12$, whereas GMW ran on circuits up to $d=23$ (about 8 million gates).
} 


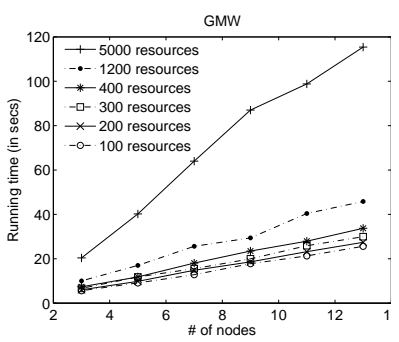

(a) GMW

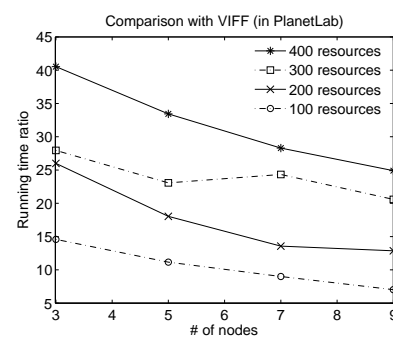

(b) $\frac{\text { VIFF }}{\text { GMW }}$

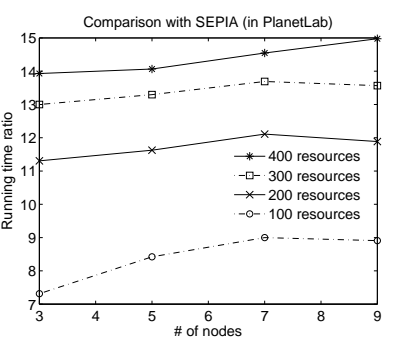

(c) $\frac{\text { SEPIA }}{\text { GMW }}$

Fig. 5. Running times in PlanetLab.

maximum round trip time (RTT) was more than $200 \mathrm{~ms}$. The test nodes in PlanetLab have various hardware specs; the least powerful node had two Intel Core2Duo 2.33GHz CPUs and 2.0GB memory, while the most powerful node had four Intel Xeon $2.83 \mathrm{GHz}$ CPUs and 3.7GB memory.

Figure 5 shows that GMW's running time increases by $17-64 \%$ relative to the time required on a LAN. (The bandwidth usage is identical whether running over a LAN or a WAN.) We also observed more variability in the running time over PlanetLab than in a LAN, which is not surprising. As we increase the number of participating nodes, the running time increases linearly (as in the LAN) even though nodes' configurations are not homogeneous; this suggests that performance is mostly affected by communication latency. GMW maintains stable performance regardless of network conditions and heterogeneous hardware configurations, consistently outperforming VIFF and SEPIA as shown in Figures 5(b) and 5(c).

\section{Conclusions}

We have shown an implementation of the GMW protocol for secure multi-party computation. Our implementation is distinguished from existing implementations of multi-party computation in two important ways: (1) Our implementation supports boolean circuits, rather than arithmetic circuits as in $[11,7]$, and (2) it provides security against a semi-honest adversary corrupting any number of parties, rather than requiring an honest majority as in $[3,11,7]$. We have also shown that our implementation outperforms previous work $[11,7]$, at least for certain classes of problems that are more amenable to being solved using boolean circuits rather than arithmetic circuits. Finally, our work shows the feasibility of applying generic secure multi-party computation to realistic networking problems where privacy is required.

\section{Acknowledgments}

Research of Jonathan Katz and Seung Geol Choi was supported by DARPA, and by NSF awards \#0447075, \#0964541, and \#1111599. Research of Tal Malkin was 
supported by a Google research grant, NSF awards \#0831094 and \#1116702, and IARPA via DoI/NBC contract number D11PC20194. Research of Dan Rubenstein and Kyung-Wook Hwang was supported by NSF award \#1017934 and DHS HSHQDC10J00204. The U.S. Government is authorized to reproduce and distribute reprints for governmental purposes notwithstanding any copyright annotation herein. The views and conclusions of this paper are those of the authors do not necessarily reflect the position or the policy of the US Government, DARPA, IARPA, or DoI/NBS, and no official endorsement should be inferred.

\section{References}

1. M. Armbrust, A. Fox, R. Griffith, A. D. Joseph, R. Katz, A. Konwinski, G. Lee, D. A. Patterson, A. Rabkin, I. Stoica, and M. Zaharia. Above the clouds: A Berkeley view of cloud computing. Technical Report UCB/EECS-2009-28, EECS Department, UC Berkeley, 2009.

2. D. Beaver. Precomputing oblivious transfer. In Advances in Cryptology Crypto '95, volume 963 of LNCS, pages 97-109. Springer, 1995.

3. A. Ben-David, N. Nisan, and B. Pinkas. FairplayMP: A system for secure multiparty computation. In 15th ACM Conf. on Computer and Communications Security, pages 257-266. ACM Press, 2008.

4. D. Bogdanov, S. Laur, and J. Willemson. Sharemind: A framework for fast privacypreserving computations. In 13th European Symposium on Research in Computer Security (ESORICS), volume 5283 of LNCS, pages 192-206. Springer, 2008. See http://sharemind.cyber.ee.

5. P. Bogetoft, D. Christensen, I. Damgård, M. Geisler, T. Jakobsen, M. Krøigaard, J. Nielsen, J. Nielsen, K. Nielsen, J. Pagter, M. Schwartzbach, and T. Toft. Secure multiparty computation goes live. In Financial Cryptography and Data Security, volume 5628 of $L N C S$, pages 325-343. Springer, 2009.

6. P. Bogetoft, I. Damgård, T. Jakobsen, K. Nielsen, J. Pagter, and T. Toft. A practical implementation of secure auctions based on multiparty integer computation. In Financial Cryptography and Data Security, volume 4107 of LNCS, pages 142-147. Springer, 2006.

7. M. Burkhart, M. Strasser, D. Many, and X. Dimitropoulos. SEPIA: Privacypreserving aggregation of multi-domain network events and statistics. In 19th USENIX Security Symposium, pages 223-240. USENIX Association, 2010.

8. R. Buyya, D. Abramson, and S. Venugopal. The grid economy. Proc. IEEE, 93(3):698-714, 2005.

9. Y. Chen, R. H. Katz, Y. H. Katz, and J. D. Kubiatowicz. Dynamic replica placement for scalable content delivery. In IPTPS, pages 306-318, 2002.

10. S.G. Choi, K. Hwang, J. Katz, T. Malkin, and D. Rubenstein. Secure multiparty computation of boolean circuits with applications to privacy in on-line marketplaces In Cryptology ePrint Archive, Report 2011/257, 2011. Available at http://eprint.iacr.org/2011/257.

11. I. Damgård, M. Geisler, M. Krøigaard, and J. Nielsen. Asynchronous multiparty computation: Theory and implementation. In 12th Intl. Conference on Theory and Practice of Public Key Cryptography - PKC 2009, volume 5443 of LNCS, pages 160-179. Springer, 2009. See http://viff.dk.

12. O. Goldreich. Foundations of Cryptography, vol. 2: Basic Applications. Cambridge University Press, Cambridge, UK, 2004. 
13. O. Goldreich, S. Micali, and A. Wigderson. How to play any mental game, or a completeness theorem for protocols with honest majority. In 19th Annual ACM Symposium on Theory of Computing (STOC), pages 218-229. ACM Press, 1987.

14. W. Henecka, S. Kögl, A.-R. Sadeghi, T. Schneider, and I. Wehrenberg. TASTY: Tool for automating secure two-party computations. In 17th ACM Conf. on Computer and Communications Security (CCCS), pages 451-462. ACM Press, 2010.

15. Y. Huang, D. Evans, J. Katz, and L. Malka. Faster secure two-party computation using garbled circuits. In 20th USENIX Security Symposium, USENIX Association, 2011.

16. Y. Ishai, J. Kilian, K. Nissim, and E. Petrank. Extending oblivious transfers efficiently. In Advances in Cryptology - Crypto 2003, volume 2729 of LNCS, pages 145-161. Springer, 2003.

17. T. Jakobsen, M. Makkes, and J. Nielsen. Efficient implementation of the Orlandi protocol. In 8th Intl. Conference on Applied Cryptography and Network Security (ACNS), volume 6123 of $L N C S$, pages 255-272. Springer, 2010.

18. J. Kangasharju, J. Roberts, and K. W. Ross. Object replication strategies in content distribution networks. In Computer Communications 25(4): 376-383, 2002.

19. V. Kolesnikov, A.-R. Sadeghi, and T. Schneider. Improved garbled circuit building blocks and applications to auctions and computing minima. In Cryptology and Network Security (CANS), volume 5888 of LNCS, pages 1-20. Springer, 2009.

20. P. R. Lewis, P. Marrow, and X. Yao. Evolutionary market agents for resource allocation in decentralised systems. In Parallel Problem Solving From Nature, volume 5199 of $L N C S$, pages 1071-1080. Springer, 2008.

21. B. Li, H. Li, G. Xu, and H. Xu. Efficient reduction of 1-out-of- $n$ oblivious transfers in random oracle model. Cryptology ePrint Archive, Report 2005/279, 2005.

22. Y. Lindell, B. Pinkas, and N. Smart. Implementing two-party computation efficiently with security against malicious adversaries. In 6th Intl. Conf. on Security and Cryptography for Networks (SCN), volume 5229 of LNCS, pages 2-20. Springer, 2008.

23. L. Malka and J. Katz. VMCrypt - modular software architecture for scalable secure computation. Available at http://eprint.iacr.org/2010/584.

24. D. Malkhi, N. Nisan, B. Pinkas, and Y. Sella. Fairplay - a secure two-party computation system. In 13th USENIX Security Symposium, pages 287-302. USENIX Association, 2004.

25. M. Naor and B. Pinkas. Computationally secure oblivious transfer. J. Cryptology, 18(1):1-35, 2005.

26. B. Pinkas, T. Schneider, N. Smart, and S. Williams. Secure two-party computation is practical. In Advances in Cryptology - Asiacrypt 2009, volume 5912 of LNCS, pages 250-267. Springer, 2009.

27. B. Schnizler, D. Neumann, D. Veit, and C. Weinhardt. Trading grid services - a multi-attribute combinatorial approach. European J. Operational Research, 187(3):943-961, 2008 .

28. Z. Tan and J. R. Gurd. Market-based grid resource allocation using a stable continuous double auction. In Proc. 8th IEEE/ACM Intl. Conf. on Grid Computing, IEEE, pages 283-290, 2007.

29. R. Wolski, J.S. Plank, J. Brevik, and T. Bryan. Analyzing market-based resource allocation strategies for the computational grid. International Journal of High Performance Computing Applications, 15(3):258-281, 2006.

30. A. C.-C. Yao. How to generate and exchange secrets. In 27th Annual Symp. on Foundations of Computer Science (FOCS), pages 162-167. IEEE, 1986. 